\title{
Nicotinic acid impairs assembly of leading edge in glioma cells
}

\author{
XIANGCAI YANG $^{1 *}$, SHUTING MEI $^{2 *}$, HUA NIU $^{1}$ and JIEJING LI ${ }^{1}$ \\ ${ }^{1}$ Department of Clinical Laboratory, The Affiliated Hospital of KMUST, Medical Faculty, \\ Kunming University of Science and Technology; ${ }^{2}$ Department of Gerontology, \\ First People's Hospital of Yunnan Province, Kunming, Yunnan 650032, P.R. China
}

Received January 9, 2017; Accepted June 15, 2017

DOI: $10.3892 /$ or.2017.5757

\begin{abstract}
Malignant glioma is a clinically formidable disease. It commonly leads to death within 5 years after diagnosis. Physicians are often baffled since the inevitable diffuse invasion deteriorates clinical outcomes rapidly. Therefore, cancerous infiltration presents a foremost challenge to all therapeutic strategies on glioblastoma multiforme (GBM). Previously, we demonstrated that nicotinic acid (NA) possesses a brand new function by targeting F-actin stress fibers. By treating HEK 293 or NIH3T3 cells with a certain concentration of NA, the F-actin stress fiber was significantly disassembled. This notable finding inspired us to explore NA further in cancer cell lines, such as GBM cells, since F-actin stress fibers are the critical foundation of cell migration, proliferation and numerous essential signaling pathways. Expectedly, we observed that optimized concentrations of NA, $3.5 \mathrm{mM}$ and $7.0 \mathrm{mM}$, detached U251 from culturing petri dishes. Moreover, $7.0 \mathrm{mM}$ of NA was capable of disrupting the leading-edge assembly. Additionally, we collected paraffin specimens from 85 GBM patients and evaluated the expression pattern of paxillin. Notably, we found that discernable paxillin signals were detected in 67 out of 85 samples. Given that leading edge is critical for cancer cell migration, we propose that NA treatment may be developed into a potential therapy for malignant glioma.
\end{abstract}

\section{Introduction}

Malignant glioma is a type of tumor that is derived from the glial cells in the nervous system. Patients commonly succumb to this deadly disease within 5 years upon being diagnosed (1).

Correspondence to: Professor Hua Niu or Dr Jiejing Li, Department of Clinical Laboratory, The Affiliated Hospital of KMUST, Medical Faculty, Kunming University of Science and Technology, Kunming, Yunnan 650032, P.R. China

E-mail:nh_ynccl@163.com

E-mail: jjli@kmust.edu.cn

*Contributed equally

Key words: glioma, nicotinic acid, leading edge, cell-matrix interaction, cytoskeleton
Glioma is classified into 4 pathological grades according to the World Health Organization (WHO). Among them, grade IV, also called glioblastoma multiforme (GBM), is recognized clinically as the most frequent and malignant category (2). Currently, therapeutic strategies involve 3 approaches, which consist of maximal tolerable surgical resection paired with radiation and chemotherapy. The combination of these therapies is capable of adding only months of additional survival. The diffuse invasion of tumor cells into the surrounding brain tissues imparts the major challenge for therapy. Although early radical surgical interventions attempt to remove the entire affected brain hemisphere, patients are usually subjugated to cancer cells that have crossed into the other hemisphere (3). Even now, with advanced microsurgical techniques, recurrence is still often inevitable. Glioma typically reoccurs within $1-2 \mathrm{~cm}$ of the primary tumor (4). Hence, a primary challenge is to prevent tumor cells from uncontrolled delamination and infiltration into other brain regions.

Cell migration is a finely tuned biological process that requires an elaborately assembled leading edge and dynamic interaction between the migrating cell and the extracellular matrix (ECM) (5). Actin-myosin molecular motors exert the main contractile stress and in particular, myosin II plays an important role in glioma invasion (6). Normally, sturdy cell attachments prevent cells from detaching and emigrating. These cell attachments are mediated by cell-cell and cell-matrix receptors, such as integrins, cadherins and neural cell adhesion molecules. Detachment of those cells entails the activation of proteases, such as matrix metalloproteinases, which dissociate tumor cells from the neighboring environment (cells and ECM) (7-9). Consequently, this promotes dynamic alterations of actin and adhesion at the cell membrane, and activation of respective downstream signaling pathways that govern adhesion and migration.

Nicotinic acid (NA), a member of the vitamin B family, is well known for its functions in the treatment and prevention of atherosclerosis. NA is one of the most effective agents capable of offering protection against cardiovascular risk factors by increasing high density lipoprotein (HDL) levels, while simultaneously decreasing very low density lipoprotein (VLDL) and low density lipoprotein (LDL) levels (10). Moreover, NA has been ascertained to function by downregulating intracellular cyclic adenosine monophosphate (cAMP), the major intracellular mediator of prolipolytic stimuli, and subsequently decreasing cellular levels of free fatty 
acids (11). Notably, another study recently indicated that NA is also able to boost intracellular calcium compartmentalization. Moreover, it further disclosed that a high concentration of NA (50 $\mathrm{mM})$ is able to co-activate TRPV1-4 transducing calcium. Concomitantly, we demonstrated that NA over $30 \mathrm{mM}$ is able to markedly disassemble the cytoskeleton and lead to the depigmentation of the Xenopus embryo (12-14).

Given the crucial function of cytoskeletal stress fibers in cell migration, our discovery of the de novo biological function of NA warrants a further investigation into daunting GBM. We initially examined distinct gradients of NA on the malignant glioblastoma cell line U251. Expectedly, we determined that both 3.5 and $7.0 \mathrm{mM}$ of NA were able to detach U251 from culture dishes. Next we investigated F-actin and $\beta$-tubulin cellular patterns and found that $7.0 \mathrm{mM}$ of NA treatment specifically abrogated the stress fiber distribution of the cytoskeleton. Supplementation with extra ECM molecules such as collagen, gelatin, poly-L-ornithine and laminin in $7.0 \mathrm{mM}$ were unable to restore cellular stress in comparison to supplementation in phosphate-buffered saline (PBS)-treated $\mathrm{U} 251$ cells. This implies that $7 \mathrm{mM}$ of NA probably modulates cytoskeletal stress via a specific biological process instead of non-specific biological toxicity. Based on these data, we propose that NA treatment may be developed into an effective therapeutic method for malignant glioma.

\section{Materials and methods}

Sources and maintenance of cells. U251 glioblastoma cell were provided by the Cell Bank of Type Culture Collection of Chinese Academy of Sciences (Shanghai, China). U251 cells were cultured in Dulbecco's modified Eagle's medium (DMEM) supplemented with $10 \%$ fetal calf serum (both from HyClone, Logan, UT, USA). Primary mouse neurons and glia were isolated from the brains of 13-15 day-old fetal ICR mice (Jackson Laboratory, Bar Harbor, ME, USA).

Primary mouse neuron/glia culture. Primary mouse neurons and glia cells were isolated from the brain of fetal ICR mice 13-15 days old. The primary mouse neurons were cultured in neurobasal media supplemented with 2\% B27 (both from Invitrogen), $0.5 \mathrm{mM}$ glutamine and $25 \mu \mathrm{M}$ glutamate (both from Sigma). The primary glia cells were cultured in the DMEM supplemented with $10 \%$ FBS. All cell lines were incubated at $37.5^{\circ} \mathrm{C}$ with $5 \% \mathrm{CO}_{2}$.

Cell viability assays. The MTT assays were carried out as described to determine the viability of U251 cells as well as normal glia and neurons (15). To perform this, cells were cultured in 96-well plates with $1 \mathrm{mg} / \mathrm{ml}$ of MTT (Amresco, LLC, Solon, OH, USA) for $4 \mathrm{~h}$. The medium was then carefully aspirated, and $100 \mu 1$ of dimethyl sulfoxide (DMSO) was added to each well to dissolve the MTT formazan crystals. The optical density was assessed at $570 \mathrm{~nm}$ with an iMark microplate absorbance reader (Bio-Rad, Hercules, CA, USA).

Immunocytochemistry. U251 cells were cultured on Lab-Tek chamber slides (Sigma-Aldrich). After treatment with PBS or NA, the cells were fixed with $4 \%$ paraformaldehyde and permeabilized with $0.4 \%$ Triton $\mathrm{X}-100$ at room temperature.
The cells were then blocked with bovine serum albumin (5\%; Amresco) and incubated with a primary antibody at $4^{\circ} \mathrm{C}$ overnight. Primary antibodies used were: $\beta$-tubulin $(1: 400 ; 556321$; BD Transduction Laboratories ${ }^{\mathrm{TM}}$, San Jose, CA, USA), p-paxillin $(1: 200 ; 44-720 \mathrm{G})$, paxillin (1:200; AHO0492), p-cortactin (1:400; 44-856) (all from Invitrogen), myosin IIA (M8064; 1:100; Sigma-Aldrich). The cells were subsequently incubated with PE or FITC-conjugated secondary antibodies (Santa Cruz Biotechnology, Santa Cruz, CA, USA) at room temperature, and labeled with DAPI (Sigma-Aldrich) to identify cell nuclei (16). F-actin stress fibers were labeled with rhodamine phalloidin $(5 \mathrm{U} / \mathrm{ml}$; R415; Invitrogen Life Technologies) in PBS for $15 \mathrm{~min}$ at room temperature as previously described (12). Fluorescence was detected using an Olympus IX81S1F-3 laser confocal scanning microscope (Olympus, Tokyo, Japan).

Western blotting. Western blot analyses for whole-cell lysates were performed using the following primary antibodies: paxillin (1:4,000), p-paxillin (1:5,000). Detection was carried out using HRP-conjugated secondary antibodies and an enhanced chemiluminescence substrate (GE Healthcare, Piscataway, NJ, USA). Membranes were stripped and reblotted for $\beta$-actin $(1: 1,000$; A5316; Sigma-Aldrich) as a loading control.

$R T$-PCR. The mRNA levels of target genes were analyzed using semi-quantitative RT-PCR. Total RNA was extracted from U251 cells with an RNA Simple Total RNA kit (Tiangen, Beijing, China), and reverse transcription was performed using the M-MLV First Strand kit (Invitrogen). Reverse transcription products were then amplified by PCR using the HotStart Taq Master Mix kit (Tiangen). Following a 'hot start' at $95^{\circ} \mathrm{C}$ for $3 \mathrm{~min}$, the samples were cycled at $95^{\circ} \mathrm{C}$ for $30 \mathrm{sec}, 55^{\circ} \mathrm{C}$ for $30 \mathrm{sec}$, and $72^{\circ} \mathrm{C}$ for $20 \mathrm{sec}$. The total number of cycles used were: 21 cycles for paxillin and 20 cycles for GAPDH. RT-qPCR was carried out with the SYBR-Green Master Mix (Thermo Fisher Scientific, Inc., Waltham, MA, USA), and samples were analyzed using a QuantStudio ${ }^{\mathrm{TM}} 7$ Flex RealTime PCR System. Following a 'hot start' at $50^{\circ} \mathrm{C}$ for $2 \mathrm{~min}$ and $95^{\circ} \mathrm{C}$ for $10 \mathrm{~min}$, the samples were then cycled at $95^{\circ} \mathrm{C}$ for $10 \mathrm{~min}, 60^{\circ} \mathrm{C}$ for $30 \mathrm{sec}$, and $72^{\circ} \mathrm{C}$ for $30 \mathrm{sec}$ for 40 cycles. The samples were then given a final 5 min extension at $72^{\circ} \mathrm{C}$. Primers used for PCR were: 5'-CTGCTGGAACTGAACGCT GTA-3' (forward) and 5'-GGGGCTGTTAGTCTCTGGGA-3' (reverse) for paxillin, and 5'-TGTGGGCATCAATGGATTT GG-3' (forward) and 5'-ACACCATGTATTCCGGGTCAAT-3' (reverse) for $G A P D H$.

Sample collection and ethics statement. All paraffin GBM samples were collected from the Affiliated Hospital of KMUST, Medical Faculty of Kunming University of Science and Technology (Kunming, China). All specimens had confirmed pathological diagnosis and were classified according to the WHO criteria.

Since these clinical materials were for research purposes only, we obtained written informed consent forms from all patients, directly. Prior consent from all patients and approval from the Ethics Committee at the Affiliated Hospital of Kunming University of Science and Technology were obtained. 


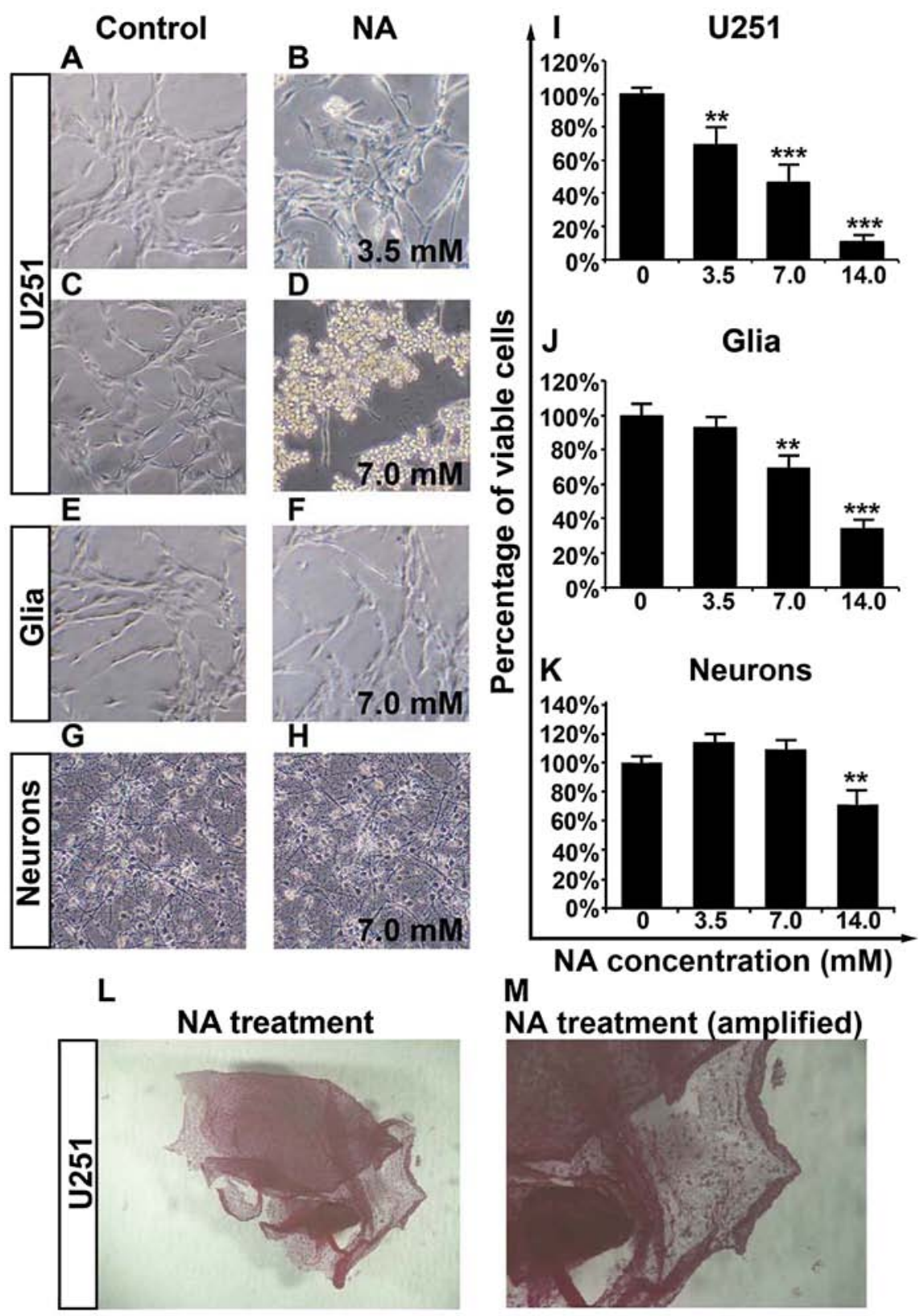

Figure 1. Overnight nicotinic acid (NA) treatment selectively detaches U251 cells and decreases their viability. (A-H) Phase contrast images of (A-D) U251 cells, (E and F) glia and (G and H) neurons that had been incubated in control (PBS) or the indicated concentration of NA for $12 \mathrm{~h}$. (I-K) Results of MTT assays displaying the viability of (I) U251 cells, (J) glia and (K) neurons treated with the indicated concentration of NA for $12 \mathrm{~h}\left({ }^{* * *} \mathrm{P}<0.01,{ }^{* * *} \mathrm{P}<0.001\right)$. PBS was used as a control ( $0 \mathrm{mM}$ of NA; same as in image below). ( $\mathrm{L}$ and M) NA treatment detaches U251 cells and preserves cell-cell adhesions, after the MTT experiment.

Methods involving live animals were carried out in accordance with the guidelines and regulations enacted and enforced by the Chinese National Ministry of Science and Technology as well as the National Ministry of Health. All experimental protocols were approved by the Institutional Laboratory Animal Ethics Committee at Kunming University of Science and Technology.

\section{Results}

Overnight treatment of U251 cells with NA causes detachment and decreased viability. We have previously reported that NA is able to modify cytoskeletal structures in 3T3 cells (12). To assess whether NA has any potential effects on malignant glioma, we carried out overnight (12-h) treatment of cultured
U251 GBM cells with various concentrations of NA. As shown in Fig. 1A-D, while PBS had some effect, incubation in NA lifted U251 cells from the culture dishes, and cells treated with $7.0 \mathrm{mM}$ of NA detached more markedly than cells treated with $3.5 \mathrm{mM}$ of NA. The detached cells remained adhered to each other to form sheet-like structures (Fig. 1L and M). Notably, 7.0 mM of NA had less impact on primary mouse glia and neurons as compared with the U251 cells (Fig. 1E-H), suggesting that the molecular target(s) of NA are abundant in GBM cells but not in primary glia or neurons.

We next employed an MTT assay and found that increasing concentrations $(3.5,7.0$ and $14.0 \mathrm{mM})$ of NA decreased the viability of U251 cells in a dose-dependent manner (Fig. 1I). Likewise, a dose-dependent decrease in the viability of primary glia was observed for NA, but the effect was weaker 


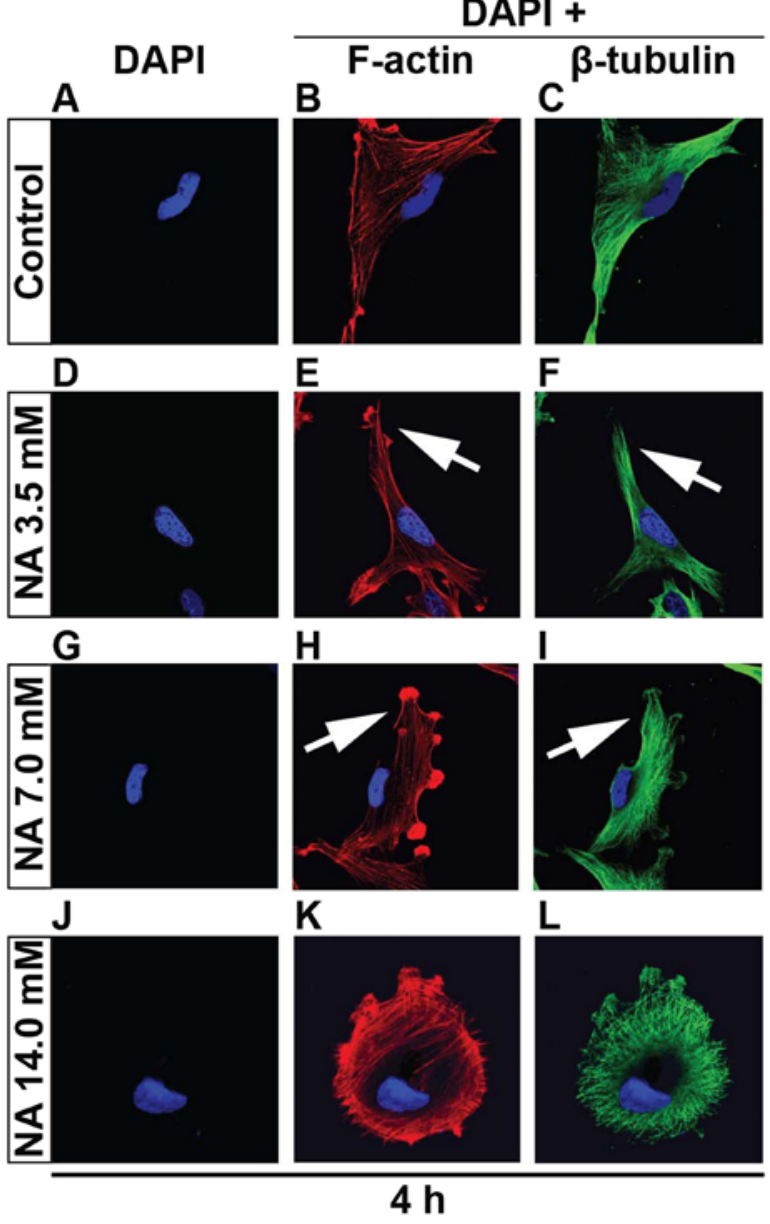

Figure 2. Nicotinic acid (NA) remodels cytoskeletal structures in U251 cells. (A and L) U251 cells were treated with the indicated concentrations of NA for $4 \mathrm{~h}$. DAPI labeling (blue) for nuclei, rhodamine phalloidin labeling for F-actin (red) and immunocytochemistry for $\beta$-tubulin (green) were carried out as described in Materials and methods. Arrows indicate disoriented stress fibers.

than on U251 cells and was insignificant at $3.5 \mathrm{mM}$ (Fig. 1J). The similar response to NA between U251 cells and normal glia could be explained by the fact that glioma cells are derived from glia and therefore share some common biological background (17). Conversely, the viability of primary neurons was only decreased by $14.0 \mathrm{mM}$, but not by lower dosages ( 3.5 and $7.0 \mathrm{mM}$ ) of NA (Fig. 1K). Hence, by carefully titrating the concentration of NA, it is possible to obtain an optimal dosage at which NA selectively targets glioma cells while sparing most normal glia and neurons.

Short-term treatment of $U 251$ cells with NA alters cytoskeletal structures. Given that overnight incubation of NA completely detaches U251 cells from the petri dish, it was necessary to cut down the time frame in order to capture the initial event. Thus we shortened the culturing time from 12 to $4 \mathrm{~h}$ since the U251 cells were still attached. That said, $14.0 \mathrm{mM}$ of NA greatly decreased the attachment of U251 cells, and tethered cells assembled (Fig. 2J-L). With gentle shakes the tethered cells detached. Again utilizing the 4-h culture time, F-actin and $\beta$-tubulin staining revealed, particularly in $7.0 \mathrm{mM}$ of NA treatment, that F-actin fibers were disoriented and entangled close to the plasma membrane (Fig. 2G-I). Likewise, $\beta$-tubulin fibers lack tension and are loosely arranged. Although $3.5 \mathrm{mM}$ of NA has a relatively minor effect on cytoskeletal stress, there were still discernable kinks in the F-actin fibers close to the plasma membrane (Fig. 2D-F). NA treatment is detrimental to cell-matrix adhesion probably due to either abrogation of components within the ECM or integrin adhesome disruption or both, since the tension exerted on adhesions requires the integrity of both ECM and integrin adhesome $(18,19)$.

Compensation of ECM components failed to restore the tension of actin fibers. The short-term effects on cytoskeletal structures and cell detachment caused by prolonged treatment, suggest that NA inhibits the adhesion of U251 cells to the matrix. In vivo, cell-matrix adhesion depends on ECM components, in particular fibrous proteins (such as collagens, fibronectins and laminins) and proteoglycans (such as chondroitin sulphate, heparan sulphate, keratan sulphate and hyaluronic acid) that are synthesized locally and meshed into an organized structure to form the fundamental framework for many tissues (20). In the context of cancer biology, increased deposition of ECM has been associated with higher mortality in cancer patients due to increased invasion and metastasis (21). It is therefore important to test whether NA also affects cell adhesion on ECM components. To do this, U251 cells were cultured on petri dishes that were pre-coated with additional collagen (Fig. 3A-F), gelatin (Fig. 3G-L), poly-Lornithine (Fig. 3M-R) or laminin (Fig. 3S-X), allowed to attach, and subsequently treated with $7.0 \mathrm{mM}$ of NA for $4 \mathrm{~h}$. Similar to what we observed previously with the unsupplemented plates, U251 cells that were cultured on plates coated with various ECM components also exhibited disoriented F-actin fibers that entangled close to plasma membrane (Fig. 3A-X). These results suggest that NA interferes with the ability of U251 cells to adhere to the ECM from an intracellular aspect, rather than directly targeting the ECM.

NA impedes the assembly of leading edge. Phosphorylation of paxillin is known to play pivotal roles in the rearrangement of the actin cytoskeletal network at focal adhesion sites, and can serve as an important indicator for active cell migration (22). Our immunocytochemistry and western blot results revealed that $\mathrm{U} 251$ cells treated with $7.0 \mathrm{mMNA}$ for $4 \mathrm{~h}$ had significantly decreased levels of phosphorylated paxillin (p-paxillin) and total paxillin when compared with untreated cells (Fig. 4A-G). In agreement with these findings, western blot analyses revealed a dose-dependent decrease of both total and phosphorylated paxillin levels upon treatment with 3.5 and $7.0 \mathrm{mM}$ of NA (Fig. 4G). However, mRNA levels of paxillin were unaffected (Fig. 4H), suggesting that NA downregulates paxillin post-transcriptionally. Phosphorylation of tyrosine residues in cortactin has also been shown to regulate focal adhesion turnover and cell migration $(23,24)$, while myosin IIA generates the major contractile forces by pulling on actin stress fibers at the trailing end of migrating cells (25-27). We found that in U251 cells incubated in $7.0 \mathrm{mM}$ of NA for $4 \mathrm{~h}$, phosphorylated cortactin (p-cortactin) markedly decreased (Fig. 5A-F), and myosin IIA dissociated from F-actin stress fibers (Fig. 5G-L). Based on these data, we conclude that short-term NA treatment can disrupt focal adhesion assembly in U251 cells. 

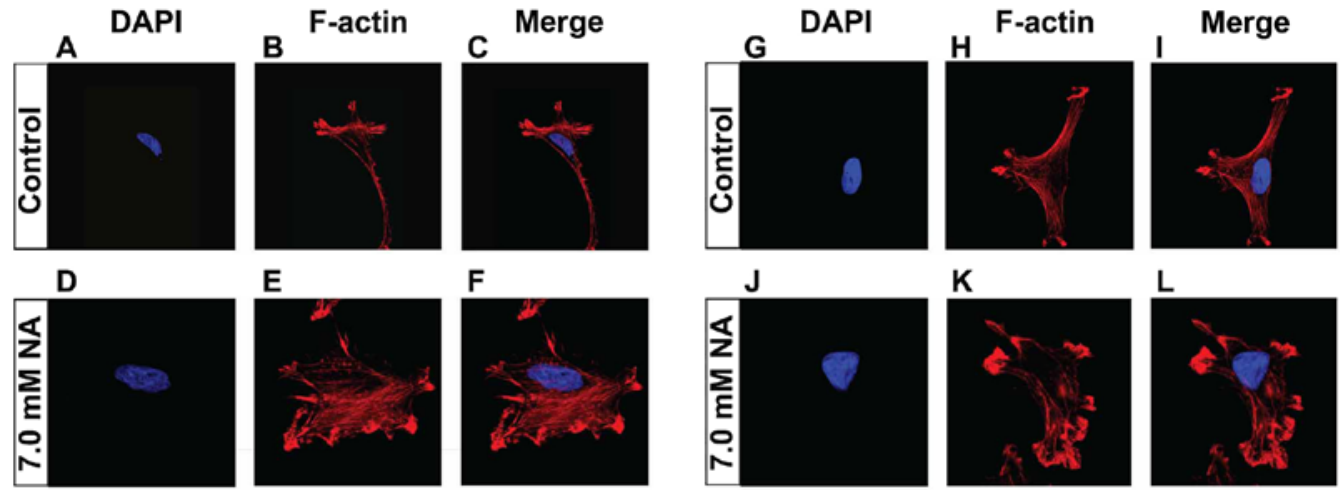

E
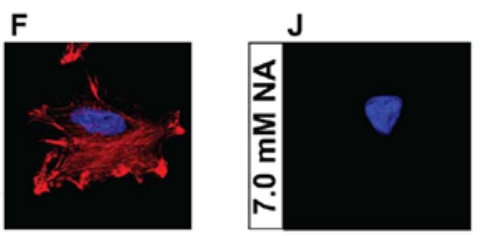

$\mathbf{K}$
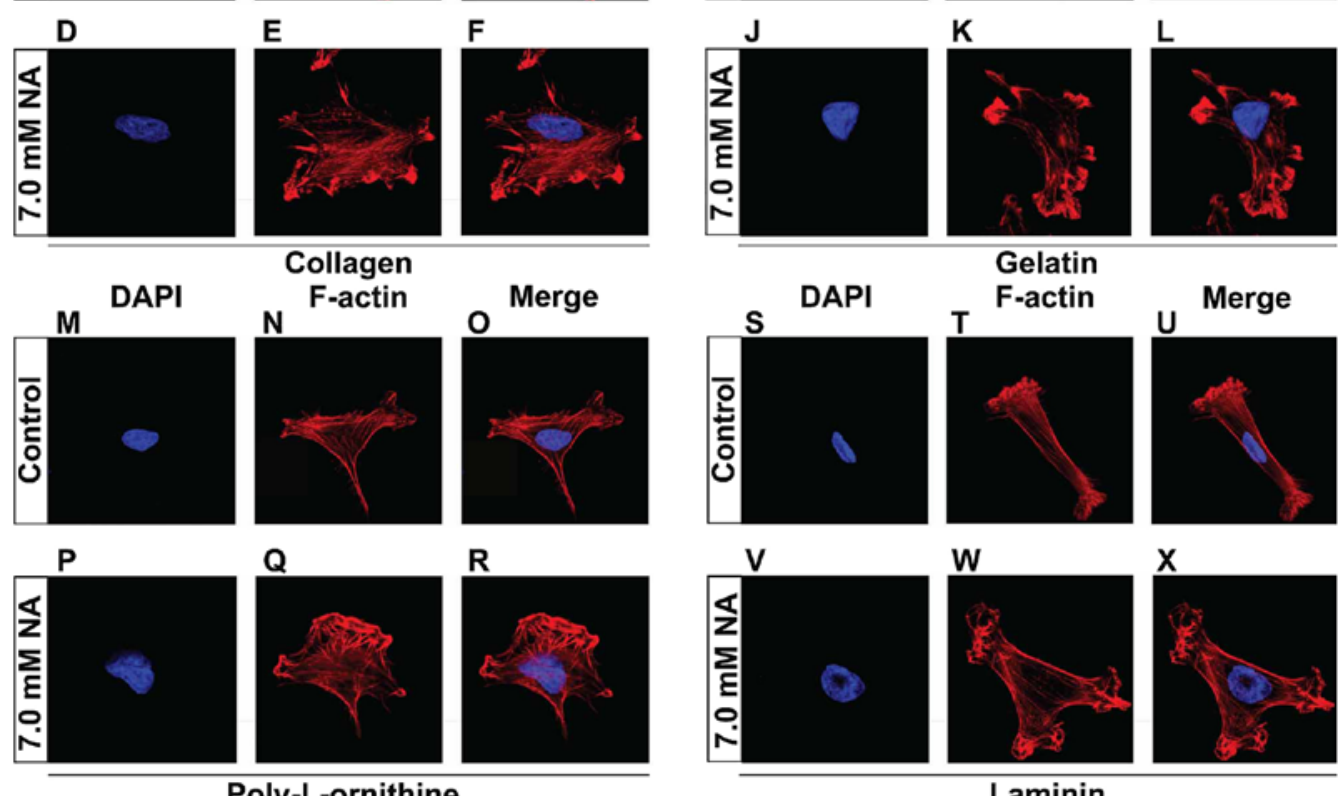

Laminin

Figure 3. Nicotinic acid (NA)-induced remodeling of cytoskeletal structures is independent of supplemented extracellular matrix (ECM) components. (A-X) U251 cells were cultured on plates coated with the indicated ECM components, and treated with the indicated concentrations of NA for 4 h. DAPI staining (blue) for nuclei, and rhodamine phalloidin labeling for F-actin (red) were carried out as described in Materials and methods.

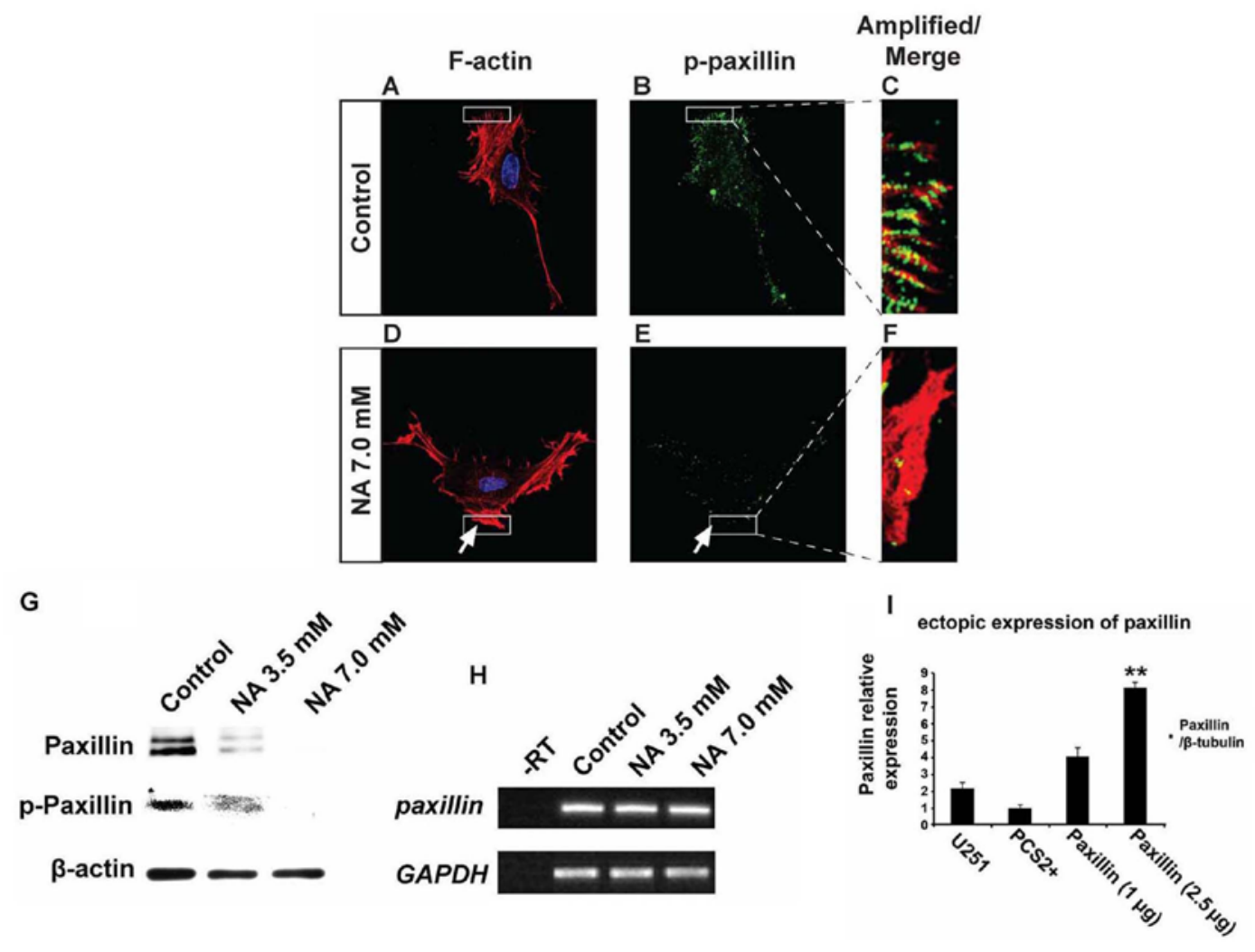

Figure 4. Nicotinic acid (NA) post-transcriptionally downregulates paxillin. U251 cells were treated with the indicated concentration of NA for $4 \mathrm{~h}$. (A-F) DAPI labeling (blue) for nuclei, rhodamine phalloidin labeling for F-actin (red) and immunocytochemistry for p-paxillin (green) were carried out as described in Materials and methods. Regions denoted with rectangles are amplified and shown in insets with red and green channels merged. (G) Western blot analyses for whole-cell lysates were performed with an anti-paxillin antibody. Membranes were stripped and reblotted for p-paxillin and $\beta$-actin. (H) Total RNA was extracted, and semi-quantitative RT-PCR was carried out for paxillin mRNA. GAPDH was included as a loading control; -RT, control with no reverse transcriptase (same below). (I) Ectopic expression of $2.5 \mu \mathrm{g}$ of paxillin in U251 cells, significantly increased paxillin mRNA. 


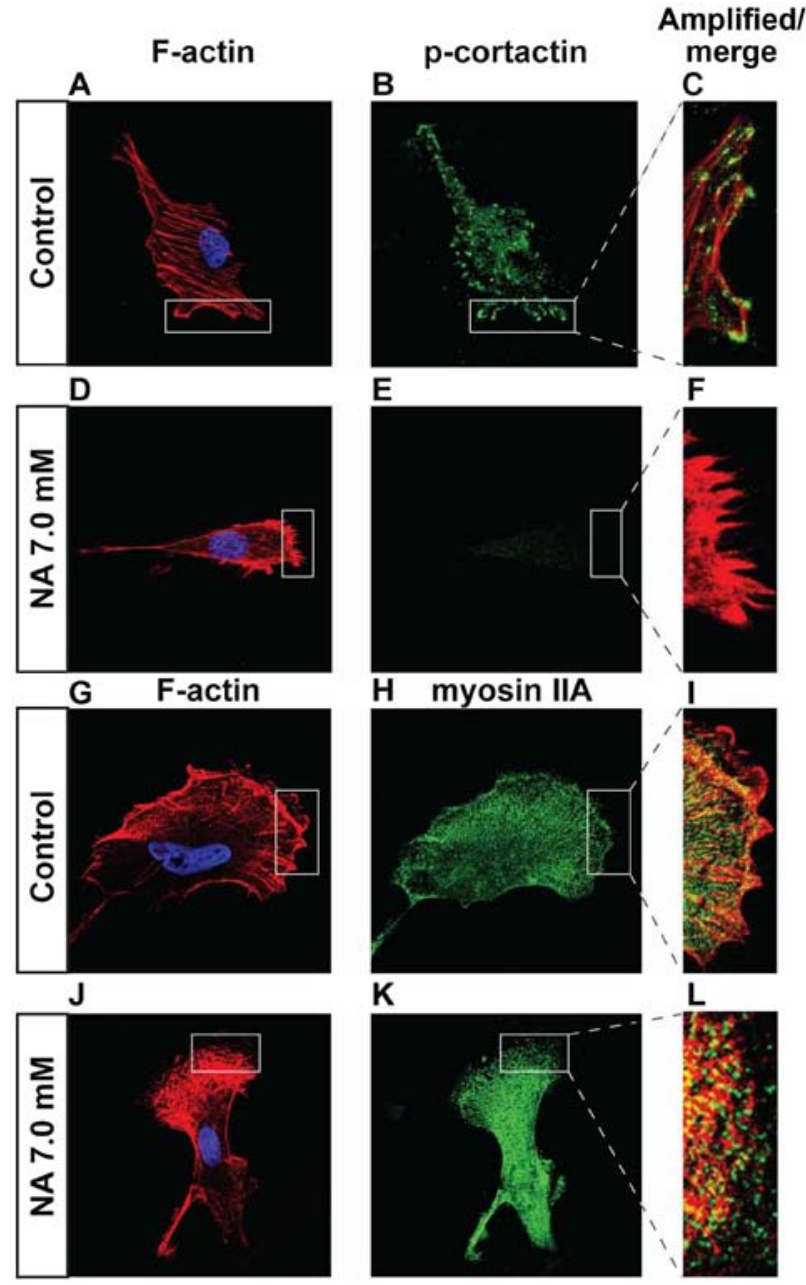

Figure 5. Effects of short-term nicotinic acid (NA) treatment on p-cortactin and myosin IIA. U251 cells were treated with the indicated concentrations of NA for $4 \mathrm{~h}$. DAPI staining for nuclei (blue) as well as rhodamine phalloidin labeling for F-actin (red in A, D, C, F and G, J, I, L), immunocytochemistry for p-cortactin (green in B, C, E and F) and myosin IIA (green in H, I, K and L) were carried out as described in Materials and methods.

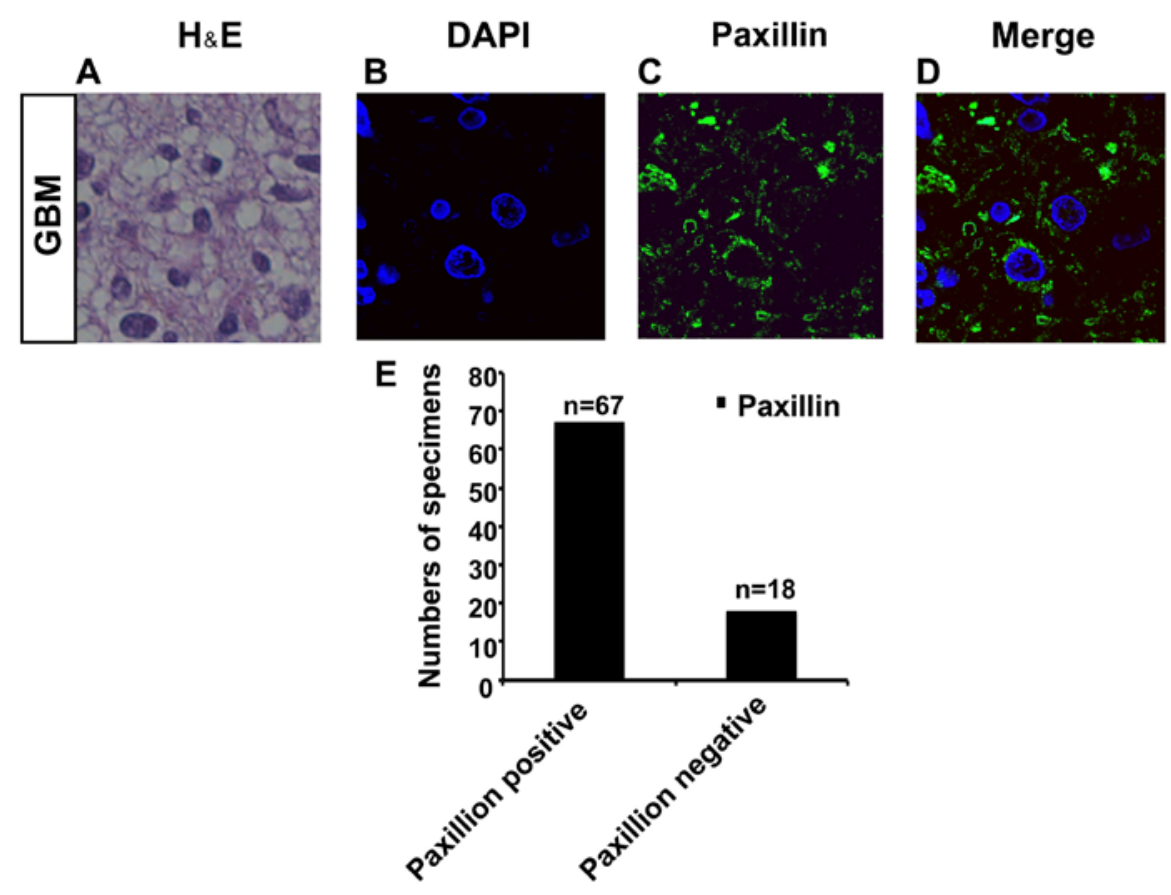

Figure 6. Paxillin is expressed in specimens of paraffin-embedded glioblastoma multiforme (GBM). (A) H\&E staining for one of the 4 paraffin-embedded GBM specimens. Paxillin is detected in 67 specimens and represented by (B-D). (E) Quantification of paxillin expression profiling in paraffin-embedded GBM specimens. 
In vivo, such disruption could lead to the inhibition of glioma cell migration by preventing firm attachment to the ECM.

Paxillin is expressed in specimens of GBM. Our immunocytochemistry and western blot analysis results showed that $4 \mathrm{~h}$ of treatment with $7.0 \mathrm{mM}$ NA significantly decreased the levels of phosphorylated paxillin (p-paxillin) and total paxillin in U251 cells in comparison to untreated U251 cells (Fig. 4A-G). This indicates that paxillin is expressed in U251 cells and is a target of NA. We therefore investigated the expression pattern of paxillin in specimens of GBM.

We collected specimens from 85 GBM patients (Fig. 6A). Interestingly, by immunostaining, we found that paxillin was expressed in 67 samples out of a total of 85 specimens (Fig. 6B-E). This corroborates that paxillin is expressed in GBM, and the findings support further investigations into NA as a potential therapy for malignant glioma.

\section{Discussion}

Although NA and its derivatives have been intensively studied for decades, new functions and mechanisms of action for them continue to emerge. For example, nicotinamide, the amide of NA that has the same vitamin functions, was shown to block proliferation and induce apoptosis of chronic lymphocytic leukemia (CLL) cells (28). Recently, we reported that treatment of 3T3 cells with NA leads to dynamic changes in intracellular calcium concentration and disassembly of the cytoskeletal structures (12). These observations prompted us to test whether NA has any effects on malignant glioma cells, which are known for their high invasive activity. We found that NA had marked effects on assembly of leading edge and cell-matrix adhesion, which suggests roles in the inhibition of glioma cell invasion. These results revealed a novel function of NA in the regulation of tumor cell migration, and set the stage for testing NA as a potential therapy for malignant glioma.

When applied overnight, NA causes detachment and decreased viability of the U251 GBM cells, effects that appear to be selective for U251 cells over normal neurons and glia (Fig. 1). Although the molecular basis for this selectivity remains unknown, our subsequent study revealed that cell death caused by NA is due to activation of the apoptotic pathway (Yang et al, unpublished data). Notably, nicotinamide has been shown to induce apoptosis in CLL cells by regulation of the sirtuin SIRT1 (28). Since SIRT1 also plays important roles in the apoptosis of malignant glioma cells (29), it may be of interest to investigate whether and how NA affects sirtuins and their downstream targets such as p53 $(30,31)$.

Unlike overnight treatment, short-term incubation of U251 cells in NA does not cause any detectable cell death, but instead impairs assembly of leading edge and inhibits cell-ECM interactions. The decrease in cell-matrix interaction is manifested by disrupting cytoskeletal structures and focal adhesion assembly. Similar to what we observed previously in 3T3 cells, NA treatment modifies F-actin and $\beta$-tubulin filaments in a dose-dependent response that correlates well with an increase in the intracellular calcium level. However, direct treatment of $3 \mathrm{~T} 3$ cells with $\mathrm{CaCl}_{2}$ alters the structures of $\mathrm{F}$-actin stress fibers but not microtubules (12), suggesting that the effect of NA on microtubules is independent of or less sensitive to the increased calcium levels. In line with the latter possibility, $3.5 \mathrm{mM}$ of NA induces subtle modifications of F-actin stress fibers, but has no detectable effect on microtubules, whereas $7.0 \mathrm{mM}$ of NA is able to remodel both structures (Fig. 2D-I). By contrast, $3.5 \mathrm{mM}$ of NA is sufficient to cause a marked decrease in the protein level of paxillin (Fig. 4G). Therefore, the disrupted focal adhesion assembly likely precedes and probably causes the alterations in the cytoskeletal structure, consistent with previous studies showing that integrin-mediated cell-matrix adhesion is important for the maintenance of both F-actin and microtubule filaments $(19,32)$.

Short-term treatment with $7 \mathrm{mM}$ of NA results in extensive disruption of focal adhesion assembly, as indicated by markedly diminished paxillin and p-cortactin as well as mislocalized myosin IIA. In particular, paxillin protein is almost completely lost (Fig. 4E-G). However, paxillin mRNA remains essentially unchanged (Fig. 4H), suggesting an efficient post-transcriptional regulation by NA. One possible explanation for these phenomena is the cleavage of paxillin by calpains, the calcium-activated proteases, which have been demonstrated to interfere with focal adhesion assembly and cell migration $(19,33)$. Indeed, we observed a dose-dependent increase of calpain activity in U251 cells treated with 3.5 and $7.0 \mathrm{mM}$ of NA (Yang et al, unpublished data). Thus, paxillin is possibly an important effector that mediates NA-induced inhibition of cell-matrix interaction. To test this hypothesis, we attempted to rescue the effects caused by NA by transfecting U251 cells with a plasmid that encodes paxillin. However, although the mRNA of exogenous paxillin was expressed at high levels (Fig. 4I), we were unable to detect any paxillin protein in transfected cells upon NA treatment (data not shown). This is in line with our observation that paxillin is effectively and post-transcriptionally downregulated by NA. Future efforts may be focused on targeting the turnover of paxillin protein, such as by blocking calpain activity. Additionally, we collected specimens from 85 GBM patients and evaluated the expression pattern of paxillin. Notably, we found that paxillin was expressed in 67 samples out of a total of 85 specimens (Fig. 6A-E).

Although this result is preliminary, it is an encouraging first step towards testing NA as a potential therapy for malignant glioma in an animal model. Since NA is widely used as an antidyslipidemic drug, the abundant clinical data that are available may greatly facilitate the trials of this vitamin in other diseases such as cancer. It should be noted, though, that we are still facing some challenges when exploring the clinical applications of NA. First of all, the mechanisms of action for NA have not been fully elucidated. In particular, it is not clear how NA is metabolized and how signals in the cells trigger the intracellular calcium spike. To this end we are investigating whether nicotinamide, which has the same vitamin function as NA but no lipid-regulating activity (34), can mimic NA in affecting cell-cell and cell-matrix adhesion. It also remains to be determined whether the cell-surface NA receptor GPR109A, which mediates the NA lipid-regulating activity, as well as the TRPV channels that have been reported to be downstream targets of NA $(10,13)$, are involved in the cellular processes that we observed for NA in the present study. Secondly, a high dosage of NA is known to cause adverse effects (34). Some of these effects, such as flushing, 
are mediated by GPR109A and TRPV channels, which may be involved in the antitumor activity of NA that we reported in the present study. Fortunately, these side-effects are not lifethreatening and may be acceptable for the treatment of highly deadly diseases such as malignant glioma. In conclusion, our data supports the potential application of NA as a therapy for malignant glioma, and further investigation is warranted into the effects of NA on other invasive tumors.

\section{Acknowledgements}

The present study was supported by the National Natural Science Foundation of China (81200878 to J.L.), and the Recruited Talent Program of KMUST (KKZ3201560014 to J.L.).

\section{References}

1. Cuddapah VA, Robel S, Watkins S and Sontheimer H: A neurocentric perspective on glioma invasion. Nat Rev Neurosci 15: 455-465, 2014

2. Louis DN, Ohgaki H, Wiestler OD, Cavenee WK, Burger PC, Jouvet A, Scheithauer BW and Kleihues P: The 2007 WHO classification of tumours of the central nervous system. Acta Neuropathol 114: 97-109, 2007.

3. Dandy WE: Removal of right cerebral hemisphere for certain tumors with hemiplegia: Preliminary report. J Am Med Assoc 90: 823-825, 1928

4. Hou LC, Veeravagu A, Hsu AR and Tse VC: Recurrent glioblastoma multiforme: A review of natural history and management options. Neurosurg Focus 20: E5, 2006.

5. Ridley AJ, Schwartz MA, Burridge K, Firtel RA, Ginsberg MH, Borisy G,Parsons JT and Horwitz AR: Cell migration: Integrating signals from front to back. Science 302: 1704-1709, 2003.

6. Beadle C, Assanah MC, Monzo P, Vallee R, Rosenfeld SS and Canoll P: The role of myosin II in glioma invasion of the brain. Mol Biol Cell 19: 3357-3368, 2008.

7. Demuth T and Berens ME: Molecular mechanisms of glioma cell migration and invasion. J Neurooncol 70: 217-228, 2004.

8. Kwiatkowska A and Symons M: Signaling determinants of glioma cell invasion. In: Glioma Signaling Springer, pp121-141, 2013. https://doi.org/10.1007/978-94-007-4719-7_7.

9. Wolfenson H, Lavelin I and Geiger B: Dynamic regulation of the structure and functions of integrin adhesions. Dev Cell 24: 447-458, 2013

10. Offermanns S: The nicotinic acid receptor GPR109A (HM74A or PUMA-G) as a new therapeutic target. Trends Pharmacol Sci 27 384-390, 2006.

11. Gille A, Bodor ET, Ahmed K and Offermanns S: Nicotinic acid: Pharmacological effects and mechanisms of action. Annu Rev Pharmacol Toxicol 48: 79-106, 2008.

12. Li J, Li Y, Zhang P, Niu H and Shi Y: Nicotinic acid modulates intracellular calcium concentration and disassembles the cytoskeleton. Mol Med Rep 10: 2805-2810, 2014.

13. Ma L, Lee BH, Clifton H, Schaefer S and Zheng J: Nicotinic acid is a common regulator of heat-sensing TRPV1-4 ion channels. Sci Rep 5: 8906, 2015.

14. Ma L, Lee BH, Mao R, Cai A, Jia Y, Clifton H, Schaefer S, $\mathrm{Xu} \mathrm{L}$ and Zheng J: Nicotinic acid activates the capsaicin receptor TRPV1: Potential mechanism for cutaneous flushing. Arterioscler Thromb Vasc Biol 34: 1272-1280, 2014

15. Sobottka SB and Berger MR: Assessment of antineoplastic agents by MTT assay: Partial underestimation of antiproliferative properties. Cancer Chemother Pharmacol 30: 385-393, 1992.
16. Qu J, Rizak JD, Li X, Li J and Ma Y: Melatonin treatment increases the transcription of cell proliferation-related genes prior to inducing cell death in C6 glioma cells in vitro. Oncol Lett 6: 347-352, 2013.

17. Camand E, Peglion F, Osmani N, Sanson M and EtienneManneville S: N-cadherin expression level modulates integrin-mediated polarity and strongly impacts on the speed and directionality of glial cell migration. J Cell Sci 125: 844-857, 2012.

18. Vicente-Manzanares M, Choi CK and Horwitz AR: Integrins in cell migration - the actin connection. J Cell Sci 122: 199-206, 2009.

19. Winograd-Katz SE, Fässler R, Geiger B and Legate KR: The integrin adhesome: From genes and proteins to human disease. Nat Rev Mol Cell Biol 15: 273-288, 2014.

20. Frantz C, Stewart KM and Weaver VM: The extracellular matrix at a glance. J Cell Sci 123: 4195-4200, 2010.

21. Gilkes DM, Semenza GL and Wirtz D: Hypoxia and the extracellular matrix: Drivers of tumour metastasis. Nat Rev Cancer 14: 430-439, 2014

22. Deakin NO and Turner CE: Paxillin comes of age. J Cell Sci 121: 2435-2444, 2008.

23. Kruchten AE, Krueger EW, Wang Y and McNiven MA: Distinct phospho-forms of cortactin differentially regulate actin polymerization and focal adhesions. Am J Physiol Cell Physiol 295: C1113-C1122, 2008.

24. Lin Y, Wu Y, Li J, Dong C, Ye X, Chi YI, Evers BM and Zhou BP: The SNAG domain of Snaill functions as a molecular hook for recruiting lysine-specific demethylase 1. EMBO J 29: 1803-1816, 2010.

25. Aguilar-Cuenca R, Juanes-García A and Vicente-Manzanares M: Myosin II in mechanotransduction: Master and commander of cell migration, morphogenesis, and cancer. Cell Mol Life Sci 71: 479-492, 2014

26. Kuo JC, Han X, Hsiao CT, Yates JR III and Waterman CM: Analysis of the myosin-II-responsive focal adhesion proteome reveals a role for $\beta$-Pix in negative regulation of focal adhesion maturation. Nat Cell Biol 13: 383-393, 2011.

27. van den Dries K, Meddens MB, de Keijzer S, Shekhar S, Subramaniam V, Figdor CG and Cambi A: Interplay between myosin IIA-mediated contractility and actin network integrity orchestrates podosome composition and oscillations. Nat Commun 4: 1412, 2013

28. Audrito V, Vaisitti T, Rossi D, Gottardi D, D'Arena G, Laurenti L, Gaidano G, Malavasi F and Deaglio S: Nicotinamide blocks proliferation and induces apoptosis of chronic lymphocytic leukemia cells through activation of the p53/miR-34a/SIRT1 tumor suppressor network. Cancer Res 71: 4473-4483, 2011.

29. Qu Y, Zhang J, Wu S, Li B, Liu S and Cheng J: SIRT1 promotes proliferation and inhibits apoptosis of human malignant glioma cell lines. Neurosci Lett 525: 168-172, 2012.

30. Sasca D, Hähnel PS, Szybinski J, Khawaja K, Kriege O, Pante SV, Bullinger L, Strand S, Strand D, Theobald M, et al: SIRT1 prevents genotoxic stress-induced p53 activation in acute myeloid leukemia. Blood 124: 121-133, 2014

31. Li L, Wang L, Li L, Wang Z, Ho Y, McDonald T, Holyoake TL, Chen W and Bhatia R: Activation of p53 by SIRT1 inhibition enhances elimination of CML leukemia stem cells in combination with imatinib. Cancer Cell 21: 266-281, 2012.

32. Byron A, Askari JA, Humphries JD, Jacquemet G, Koper EJ, Warwood S, Choi CK, Stroud MJ, Chen CS, Knight D, et al: A proteomic approach reveals integrin activation state-dependent control of microtubule cortical targeting. Nat Commun 6: 6135, 2015.

33. Cortesio CL, Boateng LR, Piazza TM, Bennin DA and Huttenlocher A: Calpain-mediated proteolysis of paxillin negatively regulates focal adhesion dynamics and cell migration. $\mathrm{J}$ Biol Chem 286: 9998-10006, 2011.

34. Offermanns S: Activation of platelet function through G protein-coupled receptors. Circ Res 99: 1293-1304, 2006. 